\title{
Terahertz Conductivity Analysis for Highly Doped Thin-Film Semiconductors
}

\author{
Aleksander M. Ulatowski ${ }^{1} \cdot$ Laura M. Herz $^{1} \cdot$ Michael B. Johnston ${ }^{1}$ iD
}

Received: 17 July 2020 / Accepted: 19 August 2020 / Published online: 15 September 2020

(C) The Author(s) 2020

\begin{abstract}
The analysis of terahertz transmission through semiconducting thin films has proven to be an excellent tool for investigating optoelectronic properties of novel materials. Terahertz time-domain spectroscopy (THz-TDS) can provide information about phonon modes of the crystal, as well as the electrical conductivity of the sample. When paired with photoexcitation, optical-pump-THz-probe (OPTP) technique can be used to gain an insight into the transient photoconductivity of the semiconductor, revealing the dynamics and the mobility of photoexcited charge carriers. As the relation between the conductivity of the material and the $\mathrm{THz}$ transmission function is generally complicated, simple analytical expressions have been developed to enable straightforward calculations of frequency-dependent conductivity from $\mathrm{THz}-$ TDS data in the regime of optically thin samples. Here, we assess the accuracy of these approximated analytical formulas in thin films of highly doped semiconductors, finding significant deviations of the calculated photoconductivity from its actual value in materials with background conductivity comparable to $10^{2} \Omega^{-1} \mathrm{~cm}^{-1}$. We propose an alternative analytical expression, which greatly improves the accuracy of the estimated value of the real photoconductivity, while remaining simple to implement experimentally. Our approximation remains valid in thin films with high dark conductivity of up to $10^{4} \Omega^{-1} \mathrm{~cm}^{-1}$ and provides a very high precision for calculating photoconductivity up to $10^{4} \Omega^{-1} \mathrm{~cm}^{-1}$, and therefore is highly relevant for studies of photoexcited charge-carrier dynamics in electrically doped semiconductors. Using the example of heavily doped thin films of tin-iodide perovskites, we show a simple experimental method of implementing our correction and find that the commonly used expression for photoconductivity could result in an underestimate of charge-carrier mobility by over $50 \%$.
\end{abstract}

Michael B. Johnston

michael.johnston@physics.ox.ac.uk

1 Department of Physics, Clarendon Laboratory, University of Oxford, Parks Road, Oxford, OX1 3PU, UK 
Keywords THz-TDS · OPTP · Thin films · Doped semiconductors $\cdot \mathrm{THz}$ conductivity analysis

\section{Introduction}

The progress of thin-film technology in photovoltaic (PV) applications has led to highly efficient solar cells employing a variety of semiconductors being developed in recent years [1]. The benefits of devices based on thin films include their ease of fabrication, wide choice of substrate materials and low cost of production [2]. One of the promising groups of materials used as sub-micron thin films are hybrid metal-halide perovskites, which exhibit excellent optoelectronic properties and are relatively easy to fabricate through solution processing using widely available precursors [3-5].

Terahertz time-domain spectroscopy (THz-TDS) and optical-pump-THz-probe (OPTP) experiments have been successfully used to investigate properties of thin films used in PV applications, by measuring the charge-carrier mobilities and the recombination dynamics of photoexcited carriers. These measurements allow one to estimate the diffusion length of electrons and holes, which directly affects the performance of semiconductor devices [6-11]. By investigating $\mathrm{THz}$ field transmission through photoexcited thin films, the type of photoconductivity can be established by comparing different conductivity models (including Drude conductivity, hopping transport, plasmon models, etc. [12]) against the $\mathrm{THz}$ transmission data. Such analysis provides valuable information about the charge transport properties in novel materials, which is essential for an improvement of their optoelectronic properties. Finally, THz-TDS on thin films in the dark (without photoexcitation) can be used as a non-contact probe of conductivity arising from doping in the semiconductors, thereby revealing directly the resistivity of the material without the need for metal contacts. The resistivity obtained in this way can be used to estimate the background dopant density when paired with charge-carrier mobility measurements.

With the fast progress of thin-film technology in photovoltaics and a rise in popularity of $\mathrm{THz}$ spectroscopy to investigate the properties of semiconducting materials, it is important to pay close attention to data analysis, in order to avoid misinterpretation of results due to artefacts $[13,14]$. In this article, we investigate the assumptions commonly made when calculating the properties of thin films from THz-TDS data, showing the limitations of the approximated expressions. We find that in highly doped thin films, with dark conductivity comparable to $10^{2} \Omega^{-1} \mathrm{~cm}^{-1}$, some of these approximations result in a significant underestimate of the photoconductivity, and therefore charge-carrier mobility in the semiconductor. Tin-iodide perovskite thin films were used as an example system, for which the assumption of negligible dark conductivity can no longer be used as it leads to significantly underestimated values of charge-carrier mobility. We provide a more precise analytical formula to be used for photoconductivity calculations in doped semiconductors, which greatly improves the accuracy of the calculated photoconductivity values, while not requiring numerical data fitting or other involved data analysis techniques, to obtain material properties from $\mathrm{THz}$ field transmission measurements. 


\section{Methodology}

\subsection{Transmission Function}

We begin by considering a common experimental method used to obtain conductivity of a material. Figure 1 shows a typical geometry used for THz-TDS measurements, in which the thin-film sample with complex refractive index $\tilde{n}_{2}=\tilde{n}$ and thickness $L$ is deposited on a thick, transparent substrate $\left(\tilde{n}_{3}=n_{3}\right)$ and placed in vacuum $\left(\tilde{n}_{1}=n_{1}=1\right)$ in the focal spot of the THz beam. A condition that is often met, and simplifies calculations is that the thickness of the substrate $D$ is large enough to allow internal reflections of the $\mathrm{THz}$ probe (of duration $t_{\mathrm{THz}}$ ) to be removed by windowing the TDS data (i.e. $\left.D>t_{\mathrm{THz}} c / 2 n_{3}\right)$ [12].

The transmitted amplitude and phase of the $\mathrm{THz}$ electric field at normal incidence through such a geometry depend on the properties of the sample, and can be calculated explicitly by considering amplitude reflection and transmission on the boundaries between media, as well as the propagation of the plane wave through the materials $[12,15,16]$,

$$
T_{\text {sample }}=t_{12} t_{23} \exp (i \tilde{n} L \omega / c) \times \mathrm{FP} \times \psi,
$$

where $\psi$ describes the propagation of the field through the substrate. The Fabry-Perot term FP due to multiple internal reflections inside the thin film is given by

$$
\mathrm{FP}=\sum_{p=0}^{\infty}\left(r_{21} r_{23} \exp (2 i \tilde{n} L \omega / c)\right)^{p}=\frac{1}{1-r_{21} r_{23} \exp (2 i \tilde{n} L \omega / c)},
$$

where $r_{i j}$ and $t_{i j}$ are the Fresnel reflection and transmission coefficients at normal incidence,

$$
t_{i j}=\frac{2 \tilde{n_{i}}}{\tilde{n_{i}}+\tilde{n_{j}}}, r_{i j}=\frac{\tilde{n_{i}}-\tilde{n_{j}}}{\tilde{n_{i}}+\tilde{n_{j}}} .
$$

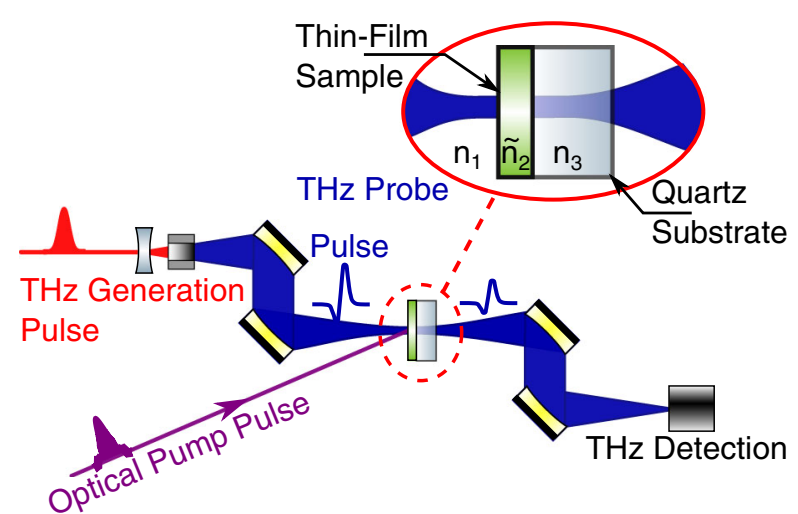

Fig. 1 Typical geometry of THz-TDS experiment, featuring terahertz pulse generation using ultrafast laser beam and a spintronic $\mathrm{THz}$ emitter, gold-coated off-axis parabolic mirrors used to focus the radiation onto the thin film and subsequently onto $\mathrm{THz}$ detector, and time-domain field detection (realised by electrooptic sampling in (110)-ZnTe crystal in our setup) 
The plane-wave approximation can be satisfied by placing the film in the focal spot of the terahertz radiation and ensuring that the sample is much thinner than the Rayleigh range of the $\mathrm{THz}$ beam [16] (usually in the range of a few millimetres). The reference transmission through the bare substrate is then given by

$$
T_{\text {substrate }}=t_{13} \exp \left(i L n_{1} \omega / c\right) \times \psi .
$$

Finally, using Eqs. 1 and 4, the relative transmission of the THz electric field through the sample can be expressed as

$$
\frac{T_{\text {sample }}}{T_{\text {substrate }}}=\frac{t_{12} t_{23}}{t_{13}} \frac{\exp \left(i\left(\tilde{n}-n_{1}\right) L \omega / c\right)}{1-r_{21} r_{23} \exp (2 i \tilde{n} L \omega / c)} .
$$

We note that the Fabry-Perot term originating from internal reflections inside the substrate was neglected in Eqs. 1 and 4, as for thick substrates the multiple internal reflections can be windowed out in time-domain measurement of the transmitted electric field [12].

\subsection{Material Properties}

The complex refractive index of a material $\tilde{n}$ depends on its polarisability, which can be described by the complex dielectric function $\tilde{\epsilon}_{r}=\epsilon_{1}+i \epsilon_{2}$. The relation between the refractive index and the dielectric function is given by

$$
\tilde{n}^{2}=\tilde{\epsilon}_{r}=\epsilon_{1}+i \epsilon_{2} .
$$

For conductive samples, one can further distinguish the individual contributions to the dielectric function from the crystal structure (through phonon modes, lattice polarisation), $\tilde{\epsilon}_{L}$, as well as the charge-carriers (movement of free charge carriers, polarisation of excitons), $i \tilde{\sigma}_{\mathrm{cc}} / \omega \epsilon_{0}$. Here, $\tilde{\sigma}_{\mathrm{cc}}$ is the complex conductivity of the charge carriers, which could be present in the semiconductor as a result of either photoexcitation or doping.

An apparent conductivity, $\tilde{\sigma}=\sigma_{1}+i \sigma_{2}$, can be then defined, which encompasses both these contributions to the overall dielectric response of the material,

$$
\tilde{\epsilon}_{r}=\tilde{\epsilon}_{L}+\frac{i \tilde{\sigma}_{\mathrm{cc}}}{\Omega \epsilon_{0}} \equiv 1+\frac{i \tilde{\sigma}}{\Omega \epsilon_{0}} .
$$

The relation between these quantities is illustrated in Fig. 2, which shows how the charge-carrier conductivity (panel A) and the dielectric function of the crystal (panel B) contribute to the overall polarisability of the sample (panel C), here presented as the apparent complex conductivity $\tilde{\sigma}$. The conductivity of charge carriers with number density $n$, mobility $\mu$ and charge $q$ was assumed to be Drude-like,

$$
\tilde{\sigma}_{\mathrm{cc}}=\sigma_{\mathrm{DC}} /(1-i \omega \tau)=n \mu q /(1-i \omega \tau),
$$

with the scattering rate of charge-carriers $1 / \tau$ comparable to $\mathrm{THz}$ frequencies. The dielectric response of the crystal is modelled by including one optical phonon mode in the presented spectral region at $\omega=\omega_{p}$, resulting in the overall dielectric function 

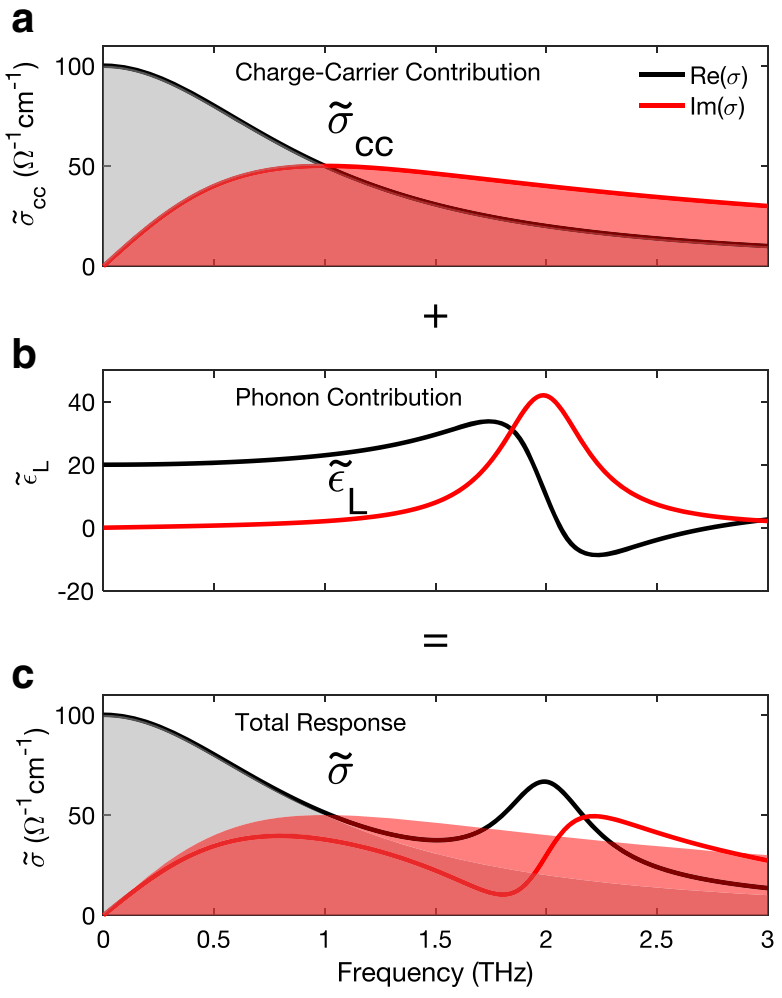

Fig. 2 a The complex conductivity of the free charge-carriers was assumed to be Drude-like (Eq. 8) with the DC conductivity of $\sigma_{\mathrm{DC}}=100 \Omega^{-1} \mathrm{~cm}^{-1}$, and the average charge-carrier scattering rate $\frac{1}{\tau}=$ $2 \pi \times 10^{12} \mathrm{~s}^{-1}$. b The dielectric contribution of the crystal is affected by the presence of an optical phonon mode at $\omega_{\mathrm{p}}=4 \pi \mathrm{THz}$, and can be described by Eq. 9. Here, the background optical dielectric constant was set to $\epsilon_{\infty}=10$, and the static dielectric constant was chosen to be $\epsilon_{\mathrm{s}}=20$. $\mathbf{c}$ The overall dielectric response was calculated from Eq. 7 and is shown as the apparent complex conductivity, with the shaded areas indicating the conductivity of the charge-carriers only, for comparison

being described by

$$
\tilde{\epsilon}_{L}=\epsilon_{\infty}-\left(\epsilon_{\mathrm{s}}-\epsilon_{\infty}\right) \frac{\omega_{\mathrm{p}}^{2}}{\omega^{2}-\omega_{\mathrm{p}}^{2}+i \omega \gamma},
$$

where $\epsilon_{\infty}$ is the high-frequency dielectric constant of the material and $\epsilon_{\mathrm{s}}$ is the static dielectric constant.

\subsubsection{Calculations}

In this publication, we use Eq. 5 to calculate numerically the complex transmission function of the THz electric field through a thin film with chosen dielectric function, $\tilde{\epsilon}_{r}$, and subsequently use it to obtain the apparent complex conductivity of the sample using commonly used approximated analytical formulas (Eqs. 10, 11 and 12). We then compare the estimated conductivity to its exact, chosen value, in order to evaluate 
the limits of these approximations. Subsequently we derive the expression shown in Eq. 17 and test it in the same way, showing much higher accuracy of the calculated photoconductivity over a large range of photo- and dark conductivity values.

We focus on the effects of background doping, which induces significant dark conductivity in samples, and model it as Drude-type conductivity throughout the article. As the effect of the phonon modes on photoconductivity calculations in the $\mathrm{THz}$ regime was previously discussed by La-o-vorakiat et al. [14], we assume for simplicity that the crystal contribution to the dielectric response can be described by an optical (high-frequency) dielectric constant. We set this dielectric constant to $\tilde{\epsilon}_{L}=\epsilon_{\infty}=10$ and do not consider phonon contribution to the conductivity spectrum in our calculations.

In common with the approach of La-o-vorakiat et al., we assess the limits of discussed approximations by comparing only the real part of the calculated conductivity to its exact values, as the imaginary part is strongly affected by experimental uncertainty, originating from variations of substrate thickness between the reference and the sample [14].

We note that although previous research investigated the limits of thin-film approximation in the context of optical determination of conductivity [17-19], the existing studies focused on the error caused by the thickness of the sample and the frequency of the probe beam, rather than the conductivity of the film. Since the thinfilm approximation can be also violated by the high refractive index of the sample [20-22], it is important to understand the effects of the complex conductivity on the validity of commonly used formulas in the regime where thin-film approximation would usually be made. In this publication, we therefore analyse the influence of complex conductivity alone on the error caused by using the over-simplified formulas for 400-nm-thick thin films probed with $\mathrm{THz}$ radiation at the frequency of $1 \mathrm{THz}$.

\section{Results}

\subsection{Dark Conductivity Calculations}

We first investigate the validity of assuming negligible optical thickness of the thin film, which is commonly made in the literature in order to calculate the conductivity of a sample from its relative THz-field-amplitude transmission obtained from THz-TDS measurements [12-15]. This condition can be written as $L \omega n / c \ll 1$ and $L \omega \kappa / c \ll 1$, where $n$ and $\kappa$ are the real and imaginary parts of the complex refractive index $\tilde{n}$, respectively. As the wavelength of the THz radiation $(\sim \mathrm{mm})$ is much longer than the thickness of the thin film $(<1 \mu \mathrm{m})$, this approximation is generally satisfied for thin films with low refractive index. As a result, Eq. 5 can be simplified and rearranged to obtain the electric field transmission equivalent of Tinkham formula [23, 24], relating the complex conductivity to the relative $\mathrm{THz}$ field transmission through the thin film [12-15],

$$
\tilde{\sigma}=\frac{T_{\text {substrate }}-T_{\text {sample }}}{T_{\text {sample }}} \frac{\epsilon_{0} c\left(n_{1}+n_{3}\right)}{L} .
$$


We assess the validity of this approximation for dark conductivity calculations in Fig. 3, which shows the relative error of the approximated value of the real part of the conductivity, compared with its exact values. As indicated by the green region in the figure, we note a high accuracy of the expression for low-conductivity materials. We find that the formula is suitable to calculate the real part of the complex dark conductivity of materials up to $10^{4} \Omega^{-1} \mathrm{~cm}^{-1}$, above which the large complex refractive index invalidates the assumption of negligible optical thickness.

We find that the large range of conductivity values for which the thin-film approximation holds well, spans a variety of materials, including inorganic-doped semiconductors [26, 27], conductive organic semiconductors [28] and even metals [29]. Equation 10 is therefore most suitable for calculating the dark conductivity of novel-doped semiconductors, provided that sub-micron thin films are used. For characterisation of highly conductive, metallic films, thinner samples (tens of nanometers) have to be used to satisfy the assumption of negligible optical thickness [30].

We also note that Eq. 10 provides a relation between the relative transmission of $\mathrm{THz}$ field through the sample and its total apparent conductivity, regardless of its origin. A semiconductor thin film could exhibit apparent conductivity in $\mathrm{THz}$ regime due to the existence of phonon modes in the investigated spectral range as well as

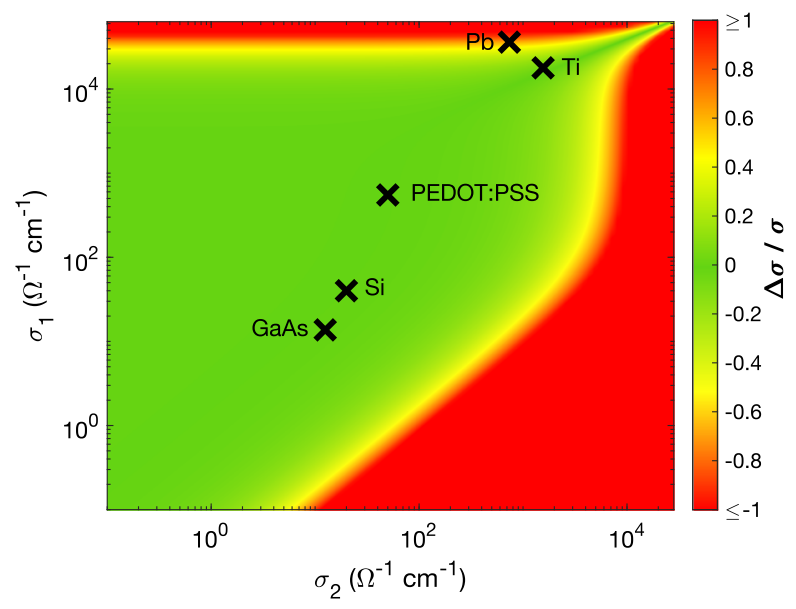

Fig. 3 The relative error $\left(\frac{\Delta \sigma}{\sigma}=\frac{\sigma_{\text {exact }}-\sigma_{\text {calculated }}}{\sigma_{\text {exact }}}\right)$ of the real part of complex apparent conductivity $\sigma_{1}$, calculated using simplified analytical expression shown in Eq. 10. The accuracy of the approximation was assessed by choosing the exact values of $\sigma_{1}$ and $\sigma_{2}$ and calculating the transmission function numerically, using Eqs. 5, 6 and 7. The calculated transmission was then used in Eq. 10 to obtain the approximated value. The calculations were performed at $v=1 \mathrm{THz}(\omega=2 \pi \mathrm{THz})$ for a 400 -nm-thick thin film deposited on a thick quartz substrate $\left(n_{3}=2.1[25]\right)$ and is shown as a function of the real and imaginary parts of the apparent complex conductivity $\tilde{\sigma}$, as defined in Eq. 7. The green region in the plot represents values of complex conductivity, for which Eq. 10 provides a good approximation and red regions indicate large deviation of calculated quantities from their exact values, as indicated by the colour bar. The positions marked with a cross represent the complex conductivities at $1 \mathrm{THz}$ of various conductive materials, including doped semiconductors and metals: $0.038 \Omega \mathrm{cm}$ n-doped GaAs[26], n-type Si with phosphorus dopant density of $10^{18} \mathrm{~cm}^{-3}$ [27], solvent-doped PEDOT:PSS (6\% DMSO)[28], titanium and lead[29] 
presence of charge carriers introduced by either electrical doping or photoexcitation. Therefore, the validity of the thin-film approximation presented in Fig. 3 is relevant to measurements of dark charge-carrier conductivity, dielectric response of the lattice, as well as the photoconductivity of the sample excited by laser pulses with above-bandgap photon energy. As such, the limits of the validity of Tinkham formula (shown in Eq. 10) discussed in this section are the upper bounds of validity limits of all formulas we assess in the following sections, all based on the assumption of negligible thickness of the sample.

\subsection{Photoconductivity: Common Approach}

We next consider the effect of a photoexcitation on the properties of the material, by modelling a uniform excitation of free charge carriers throughout the thickness of the thin film. Such uniform photoexcitation is achieved when the absorption depth at the wavelength of the pump beam (used to photoexcite the film, as shown in Fig. 1) is much longer than the thickness of the sample, which can be easily realised by exciting the semiconductor with pump photon energy just above the bandgap energy or by using sufficiently thin samples. We assume that the photoexcited charge carriers exhibit Drude-like electric conductivity (which is typical for three-dimensional bulk inorganic semiconductors at room temperature) and we check the validity of the approximations at a probe frequency of $1 \mathrm{THz}$ for very short charge-carrier scattering time $(\tau \ll 1 \mathrm{ps})$. Finally, in the absence of free charge carriers, we assume that the dielectric response of the films can be described by a constant real dielectric function (here we use $\epsilon_{L}=10$ ), which is a good assumption for materials without strong phonon mode response in the $\mathrm{THz}$ spectral region. To put the values of photoconductivity used in this section into the context of experimental parameters, we estimate that for a material with sum charge-carrier mobility of $100 \mathrm{~cm}^{2} \mathrm{~V}^{-1} \mathrm{~s}^{-1}$, in order to achieve DC photoconductivity of $100 \Omega^{-1} \mathrm{~cm}^{-1}$, charge-carrier density of around $6 \times 10^{18} \mathrm{~cm}^{-3}$ has to be photoexcited (see Eq. 8). In a 400-nm-thick thin film, uniformly photoexcited by a pulsed laser with wavelength of $800 \mathrm{~nm}$, this charge-carrier density corresponds to a photoexcitation laser pulse (pump pulse) fluence of $\sim 60 \mu \mathrm{J} / \mathrm{cm}^{2}$, assuming that all photons are absorbed and that each photon photoexcites one electron-hole pair.

The photoconductivity of a thin film, which is not conductive in dark, can be calculated from an expression similar to that for the dark conductivity, given in Eq. 10, but using the unexcited sample as a reference,

$$
\tilde{\sigma}_{\text {photo }}=-\frac{\Delta T}{T+\Delta T} \frac{\epsilon_{0} c\left(n_{1}+n_{3}\right)}{L},
$$

where $\Delta T$ is the transient electric-field-transmission change between the photoexcited thin film and the reference sample in the dark $\left(T_{\text {pump-on }}-T_{\text {pump-off }}\right)$ and $T$ is the electric field transmission through the sample in the dark $T=T_{\text {pump-off. For very }}$ small changes in transmission $(\Delta T \ll T)$, caused by low photoconductivity of the sample, Eq. 11 can be further approximated as [13]

$$
\tilde{\sigma}_{\text {photo }}=-\frac{\Delta T}{T} \frac{\epsilon_{0} c\left(n_{1}+n_{3}\right)}{L},
$$


an expression which is commonly used to calculate the photoconductivity of thin films from $\mathrm{THz}$ transmission measurements [6, 7, 9, 10, 31-33].

Figure 4 shows the deviation of the real part of the photoconductivity value calculated using Eq. 11 from the value chosen to calculate the $\mathrm{THz}$ transmission function for thin films with different values of dark conductivity. We note that the presence of free charge carriers in the unexcited sample (used as reference) significantly affects the accuracy of the expression, even for purely real values of charge-carrier conductivity (Drude with scattering time $\tau \ll 1 / \omega_{\mathrm{THz}}$ ). However, the linearity of the calculated photoconductivity with its exact value indicates that only the proportionality constant between the photoconductivity and $\frac{\Delta T}{T+\Delta T}$ term in Eq. 11 is affected by the presence of dark conductivity. We also note that the assumption of a small change in transmission, used to derive Eq. 12, is invalid for photoconductivity values above $\sim 10 \Omega^{-1} \mathrm{~cm}^{-1}$, as indicated by the red dotted line in the inset of Fig. 4. The inset shows the relative error of the photoconductivity calculated from Eqs. 11 and 12.

We conclude that Eq. 11 is suitable for photoconductivity estimates in the case of non-conductive thin films and that it provides a highly accurate value, even for high excitation fluences. We note that Eq. 12 can be used only for sufficiently low

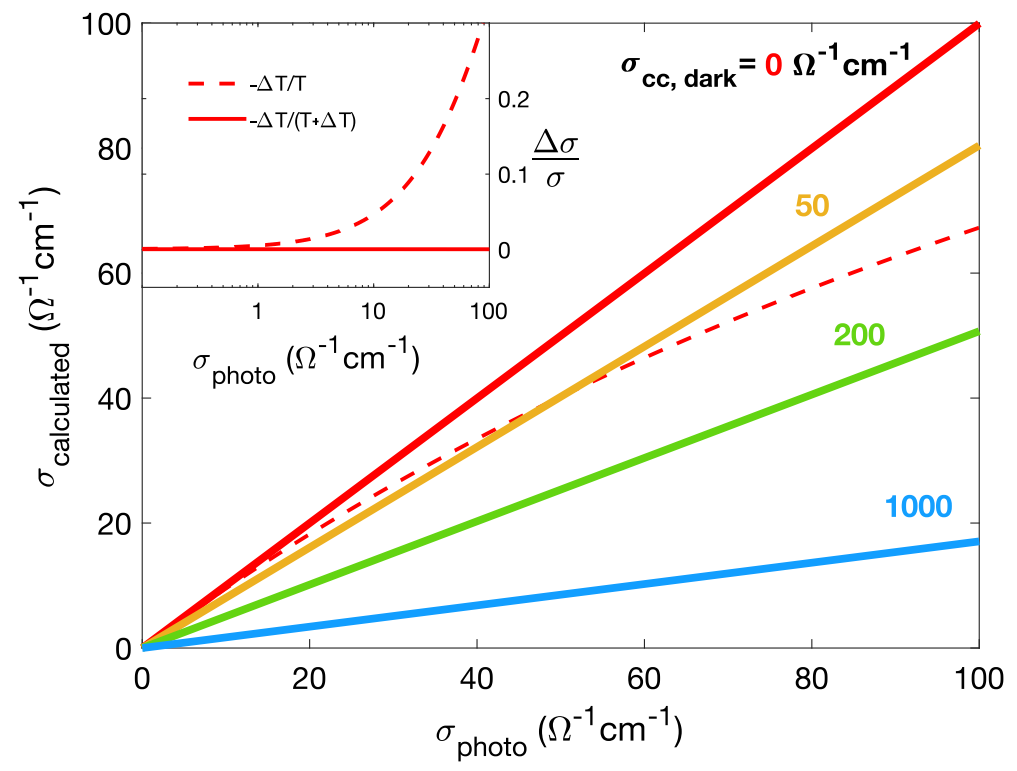

Fig. 4 The deviation of the real part of photoconductivity calculated using Eq. $11\left(\sigma_{\text {calculated }}\right)$ from its exact (chosen) value $\left(\sigma_{\text {photo }}\right)$, obtained for a few different values of dark charge-carrier conductivity $\left(\sigma_{\mathrm{cc}, \text { dark }}\right.$, indicated in $\Omega^{-1} \mathrm{~cm}^{-1}$ in the colour of the relevant solid line). The red dotted line represents the photoconductivity at $\sigma_{\mathrm{cc} \text {, dark }}=0$, calculated assuming negligible relative change of THz transmission between excited and unexcited films (Eq. 12). The inset shows the relative error $\Delta \sigma / \sigma=\left(\sigma_{\text {photo }}-\sigma_{\text {calculated }}\right) / \sigma_{\text {photo }}$ of the photoconductivity values obtained from Eq. 11 (solid line) and Eq. 12 (dotted line) for dark conductivity $\sigma_{\text {dark }}=0$. The accuracy of the approximate expressions was calculated for the frequency of $1 \mathrm{THz}$ $(\omega=2 \pi \mathrm{THz})$ for a $400-\mathrm{nm}$-thick thin film deposited on a thick quartz substrate $\left(n_{3}=2.1\right)$. The dielectric constant of $\epsilon_{L}=10$ was used in the calculations to represent polarisation effect of the crystal lattice and the photoconductivity and dark conductivity due to free charge-carriers in the film were assumed to be real (DC Drude conductivity) 
photoconductivities, lower than $10 \Omega^{-1} \mathrm{~cm}^{-1}$ in the case of $400-\mathrm{nm}$-thick films. However, neither of the equations discussed above provides enough accuracy for calculating the photoconductivity of doped semiconductors, as has been previously pointed out by Burdanova et al. [34]. These approximations assume a lack of conductivity of the unexcited sample, which is invalid in doped semiconductors. We instead suggest that the analytical expression for photoconductivity given by Eq. 17 is more appropriate in the case of conductive thin films, as discussed in the following section.

\subsection{Photoconductivity of Conductive Films}

\subsubsection{Derivation}

Using the approach presented by Nienhuys and Sundström [13], we derive a simple analytical expression for calculating the photoconductivity of highly doped semiconducting thin films from THz-TDS data. We start from the time-domain relation between the electric current density $J(t)$ flowing through a photoexcited thin film $(L \rightarrow 0)$ and the electric field incident $\left(E_{\mathrm{in}}(t)\right)$ and transmitted $\left(E_{\mathrm{tr}}(t)\right)$ through the film [13],

$$
J^{\text {pump-on }}(t)=\frac{\epsilon_{0} c}{L}\left[2 n_{1} E_{\mathrm{in}}-\left(n_{1}+n_{3}\right) E_{\mathrm{tr}}^{\text {pump-on }}\right],
$$

where we consider the thin film to be placed on the boundary between two media with refracting indices $n_{1}$ and $n_{3}$, respectively (as illustrated in Fig. 1). For a highly doped semiconducting thin film, the current density flowing through the unexcited sample is then

$$
J^{\text {pump-off }}(t)=\frac{\epsilon_{0} c}{L}\left[2 n_{1} E_{\text {in }}-\left(n_{1}+n_{3}\right) E_{\mathrm{tr}}^{\text {pump-off }}\right] .
$$

Combining Eqs. 14 and 13, and using a Fourier transform to obtain the frequencydependent current density $\tilde{J}(\omega)$ and transmitted electric field $T(\omega)$ results in

$$
\tilde{J}^{\text {pump-on }}(\omega)=\tilde{J}^{\text {pump-off }}(\omega)+\frac{\epsilon_{0} c\left(n_{1}+n_{3}\right)}{L}\left[T_{\text {pump-off }}-T_{\text {pump-on }}\right],
$$

where $T_{\text {pump-on }}$ and $T_{\text {pump-off }}$ are the frequency-dependent $\mathrm{THz}$ electric field transmission functions through the photoexcited film and film in the dark, respectively. Using the definition of complex conductivity of the thin film, $\tilde{J}^{\text {pump-on }}(\omega)=$ $\tilde{\sigma}_{\text {pump-on }} T_{\text {pump-on }}$ and $\tilde{J}^{\text {pump-off }}(\omega)=\tilde{\sigma}_{\text {dark }} T_{\text {pump-off }}$, leads to

$$
\tilde{\sigma}_{\text {pump-on }} T_{\text {pump-on }}=\tilde{\sigma}_{\text {dark }} T_{\text {pump-off }}+\frac{\epsilon_{0} c\left(n_{1}+n_{3}\right)}{L}\left[T_{\text {pump-off }}-T_{\text {pump-on }}\right],
$$

where $\tilde{\sigma}_{\text {pump-on }}$ is the total apparent conductivity of the photoexcited sample and $\tilde{\sigma}_{\text {dark }}$ is the dark conductivity of the film without photoexcitation, including the lattice conductivity.

Equation 16 can be rearranged to obtain the photoconductivity $\tilde{\sigma}_{\text {photo }}$, which is the difference of the total apparent conductivity of the photoexcited sample $\tilde{\sigma}_{\text {pump-on }}$ and apparent dark conductivity of the film $\tilde{\sigma}_{\text {dark }}$,

$$
\tilde{\sigma}_{\text {photo }}=\tilde{\sigma}_{\text {pump-on }}-\tilde{\sigma}_{\text {dark }}=\left[\tilde{\sigma}_{\text {dark }}+\frac{\epsilon_{0} c\left(n_{1}+n_{3}\right)}{L}\right] \frac{T_{\text {pump-off }}-T_{\text {pump-on }}}{T_{\text {pump-on }}} .
$$




\subsubsection{Assessment}

Figure 5 shows the comparison of the photoconductivity calculated using Eq. 17 with its exact value, and demonstrates the very high accuracy of our analytical expression, even for high values of dark conductivity and photoconductivity. The calculation was performed for thin films with Drude-type conductivity with very short charge-carrier scattering time (here the limit $\tau \rightarrow 0$ was used). Figure 7 in the Appendix further assesses our analytical formula for a few different charge-carrier scattering times, ranging between $2 \pi \tau=0.01 \mathrm{ps}$ and $2 \pi \tau=10 \mathrm{ps}$, and shows that the approximation yields a highly accurate value of photoconductivity for dark conductivity and photoconductivity values as high as $10^{4} \Omega^{-1} \mathrm{~cm}^{-1}$.

We note that our analytical formula, given by Eq. 17, provides especially high accuracy of the calculated photoconductivity for semiconductors with short
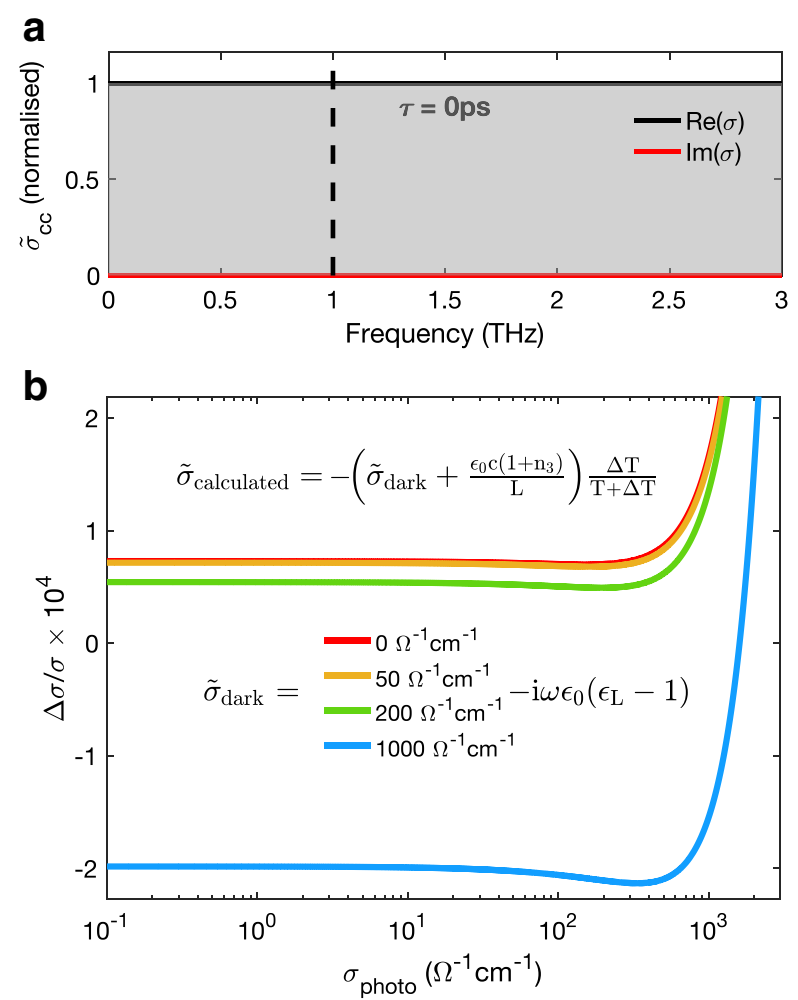

Fig. 5 a Charge-carrier conductivity spectrum used for the assessment of Eq. 17 for photoconductivity calculations. As illustrated in the figure, for Drude conductivity with very short charge-carrier scattering times, the conductivity due to carriers is purely real and spectrally flat. b The accuracy of the analytical expression for photoconductivity of highly doped thin films, shown in the figure and in Eq. 17, calculated as the relative error of the real part of photoconductivity $\Delta \sigma / \sigma=\left(\sigma_{\text {photo }}-\sigma_{\text {calculated }}\right) / \sigma_{\text {photo }}$, similarly to the inset in Fig. 4. The deviation was calculated for a 400-nm-thick thin film with dielectric constant of $\epsilon_{L}=10$ in the absence of free charge carriers, deposited on quartz, at frequency of $1 \mathrm{THz}$ (indicated in panel A with black dotted line) 
charge-carrier scattering time (and hence purely real charge-carrier conductivity), as evident from Figs. 5 as well as 7 in the Appendix. Such short carrier scattering times have been observed in perovskite semiconductors [7], which together with the reported high dark conductivity values of tin-based perovskites $\left(\sim 150 \Omega^{-1} \mathrm{~cm}^{-1}\right)$ [35] makes our approach particularly relevant to this field.

\section{Experimental Implementation}

In this section, we discuss a simple experimental technique of estimating the apparent dark conductivity, necessary to calculate the time resolved photoconductivity of doped thin films in a optical-pump-THz-probe study. As discussed by Zhao and Chia [33], OPTP measurement is a 1D THz-TDS scan, in which the magnitude of a single-cycle $\mathrm{THz}$ pulse is measured at its peak for different pump-probe time delays. The measurement provides a frequency-averaged, time-dependent real part of the photoconductivity [33] of the sample.

In such experiment, Eq. 17 can be used for photoconductivity calculations, with $\sigma_{\text {dark }}$ estimated from relative $\mathrm{THz}$ transmission through the sample in the dark, as shown in Eq. 10. In this equation, the electric field transmission through the sample $T_{\text {sample }}$ is equal to the transmission in the dark in OPTP measurements, $T_{\text {dark }}=T$, leading to

$$
\tilde{\sigma}_{\text {photo }}=-\left[\tilde{\sigma}_{\text {dark }}+\frac{\epsilon_{0} c\left(n_{1}+n_{3}\right)}{L}\right] \frac{\Delta T}{T+\Delta T}=-\frac{T_{\text {substrate }}}{T}\left[\frac{\epsilon_{0} c\left(n_{1}+n_{3}\right)}{L}\right] \frac{\Delta T}{T+\Delta T} .
$$

Figure 6 shows the experimental transmission function of a $\mathrm{THz}$ pulse through perovskite thin films of different compositions (shown in coloured solid lines) and through a quartz substrate reference (shown by the black dotted line). We note that the field strength of the transmitted pulse through tin-iodide perovskite (shown in red) is significantly reduced compared to lead-iodide perovskite (green). This difference in magnitude is caused by background hole doping present in tin-based perovskites $[35,36]$, which increases significantly the conductivity of the film in the dark. Upon addition of tin-fluoride to tin perovskite during deposition (shown to reduce the background doping[35, 37]), the THz electric field transmission through the film increases (indicated by the yellow solid line), as expected for a less conductive sample.

The inset in the figure shows the real part of the Fourier-transformed transmission functions in the frequency space, shown in the colour corresponding to the relevant transmissions in the time domain. These data are presented as the relative transmission $\frac{T_{\text {reference }}(\omega)-T_{\text {sample }}(\omega)}{T_{\text {sample }}(\omega)}$, which is proportional to the real part of dark conductivity (see Eq. 10). We note that the relative transmission in the presented spectral region is relatively spectrally flat, owing to very short charge-carrier scattering time reported for these materials, about $1.7 \mathrm{fs}$ [35]. According to Drude model of conductivity (Eq. 8), the characteristic frequency (frequency for which the real part of conductivity decreases from its maximum DC value by a factor of 2) for such a material is of the order of $100 \mathrm{THz}$. As this frequency lies in the mid-IR range of the electromagnetic 
spectrum, unreachable by most $\mathrm{THz}$ spectroscopy setups, the THz-TDS measurement in transmission mode probes directly the DC conductivity of perovskites (and similar materials with fs charge-carrier scattering time).

We next estimate the real dark conductivity of the thin films using only the magnitude of the transmitted $\mathrm{THz}$ pulse at its peak position, and plot the quantity $\frac{\max \left(E_{\text {reference }}(t)\right)-\max \left(E_{\text {sample }}(t)\right)}{\max \left(E_{\text {sample }}(t)\right)}$ in the inset of Fig. 6 in dotted lines. Since the conductivity calculated from the peak of the transmitted time-domain $\mathrm{THz}$ pulse closely follows the frequency-averaged real part of the frequency-dependent conductivity (weighted by the power spectrum of the probe-see Section A.2 in the Appendix), we expect that the plotted quantity would approximate the spectrally flat conductivity of perovskites well. Indeed, we find good agreement between our estimate and the frequency-averaged dark conductivity of the samples and recognise that this method of determining the real dark conductivity is sufficient to be used in OPTP measurements for samples with relatively short charge-carrier scattering time. Equation 18 can therefore be used with the $\frac{T_{\text {substrate }}}{T}$ term evaluated at the peak of the $\mathrm{THz}$ pulse transmitted through the substrate and the sample, without the need for calculating the dark conductivity explicitly to account for its influence on photoconductivity.

We appreciate that although our method provides very good accuracy when estimating frequency-averaged, real part of photoconductivity in OPTP experiment of semiconductors with short charge-carrier scattering time, more careful treatment is necessary when calculating the photoconductivity spectrum of samples with strong

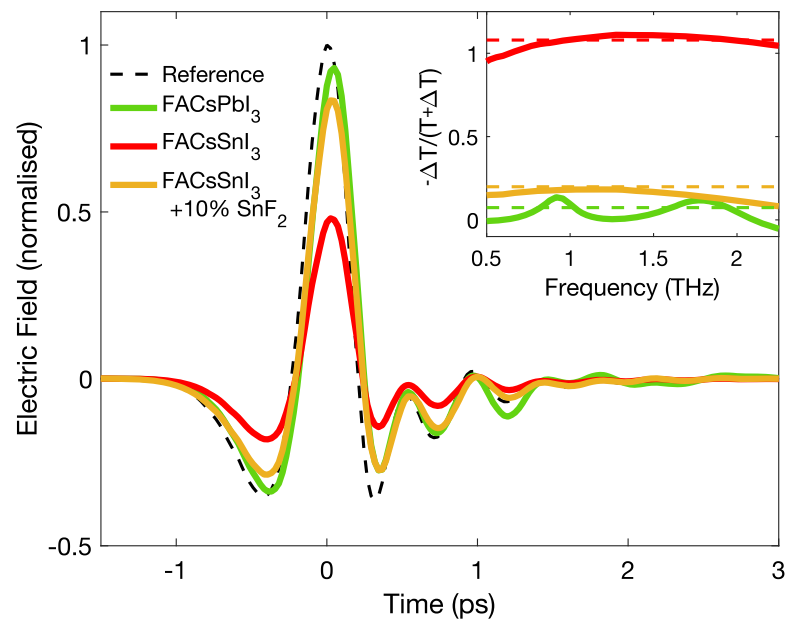

Fig. 6 The dependence of time-domain $\mathrm{THz}$ field transmission through perovskite thin films on the chemical composition of the materials. The films of lead-based (green) and tin-based (red and yellow) metal-halide perovskites were deposited on quartz substrate. The field transmitted through tin-iodide perovskite with $10 \%$ tin fluoride added to the precursor solution is shown in yellow. Dotted black line represents the field transmitted through the bare substrate. All transmission functions were normalised with respect to the transmission through the quartz substrate reference, whose magnitude was set to 1 at the peak of THz pulse. The inset shows in solid lines the real part of relative complex transmission change, $\frac{-\Delta T}{T+\Delta T}=\frac{T_{\text {reference }}-T_{\text {sample }}}{T_{\text {sample }}}$, proportional to the real dark conductivity of the film. The dotted lines represent the relative transmission calculated from the field strength at the peak of the $\mathrm{THz}$ pulse 
resonances in the $\mathrm{THz}$ spectral region [14]. To calculate the photoconductivity of such thin films, numerical fitting of the data using Eq. 5 might be necessary [16], although in some cases one can follow the treatment presented by La-o-vorakiat et al. [14]. We also find that estimating the dark conductivity from the time-domain $\mathrm{THz}$ peak leads to a significant underestimate of the DC Drude conductivity in semiconductors with relatively long scattering times $(2 \pi \tau>1 \mathrm{ps}$, which includes inorganic semiconductors such as Si and GaAs), as discussed in the Appendix; therefore, spectral analysis of dark conductivity has to be performed in these materials. Our method, however, can be successfully used when investigating materials with short scattering times, making it particularly relevant to OPTP measurements of perovskite thin films and doped organic semiconducors.

Finally, we evaluate the relative error of photoconductivity value calculated from Eq. 11 (caused by the assumption of negligible dark conductivity), using the example of tin-iodide perovskite $\mathrm{FACsSnI}_{3}$. TDS data of this perovskite thin film is presented in Fig. 6 in red solid line. We use Eq. 18 to calculate the exact value of photoconductivity $\sigma_{\text {exact }}$ (which provides a very good approximation as discussed in Section 3.3) and compare it with the approximated value $\sigma_{\text {approx }}$, calculated from Eq. 11. This results in the relative error of photoconductivity $\sigma$ (which due to the proportionality shown by Eq. 8 is also the relative error of calculated charge-carrier mobility $\mu$ ) given by

$$
\frac{\Delta \mu}{\mu}=\frac{\Delta \sigma}{\sigma}=\frac{\sigma_{\text {approx }}-\sigma_{\text {exact }}}{\sigma_{\text {exact }}}=\frac{T}{T_{\text {substrate }}}-1 \simeq-0.52,
$$

where $T$ represents the $\mathrm{THz}$ field transmission through the sample in the dark, here estimated from the peak of time-domain electric field transmission, shown in Fig. 6 in red solid line. We note that, for this tin-iodide perovskite thin-film sample, assuming negligible dark conductivity and using Eq. 11 for the calculation of photoconductivity would result in a large, $52 \%$ underestimate of photoconductivity (and therefore charge-carrier mobility), compared to its true value. This shows the significance of adapting more precise data analysis techniques, through either numerical data fitting or more precise approximations (such as Eqs. 17 and 18) when investigating properties of conductive thin films.

\section{Conclusions}

We have assessed the validity of approximations commonly used to calculate conductivity of thin films from THz-TDS data. We found that the simple analytical expression for complex conductivity, shown by Eq. 10, provides a highly accurate approximation of the real dark conductivity of thin films with low dielectric constant, up to conductivity values of the order of $10^{4} \Omega^{-1} \mathrm{~cm}^{-1}$. However, when calculating the photoconductivity of highly doped thin films (with conductivity comparable to $\sim 100 \Omega^{-1} \mathrm{~cm}^{-1}$ ), the expression commonly used in the literature (Eq. 11) results in a significant deviation of the calculated quantity from its exact value, leading to an underestimation of photoconductivity and hence the charge-carrier mobility.

We present a simple correction to the analytical expression (shown in Eqs. 17 and 18) that eliminates this deviation and can be used for photoconductivity calculations 
of doped semiconductors, within the regime of optically thin films (up to a conductivity value of the order of $10^{4} \Omega^{-1} \mathrm{~cm}^{-1}$ ). We find a simple experimental method of implementing our correction in semiconductors with sub-picosecond chargecarrier scattering times, which can be used for 1D photoconductivity scans in OPTP experiment, providing the frequency-averaged, real part of the photoconductivity, accounting for even very high background dark conductivity of the material.

Our study gives practical insights into limitations of commonly used assumptions when calculating conductivity of thin films from THz-TDS data, and provides an approximation yielding highly accurate values of photoconductivity in doped semiconductive thin films. These results are extremely relevant when investigating properties of novel materials, allowing much higher precision of photoconductivity calculations without the need for numerical fitting of data. We hope that our investigation can be used as a guide for choosing the best-suited data analysis method for a variety of THz-TDS studies.

Funding The authors received financial support from Engineering and Physical Sciences Research Council (EPSRC), UK., through research grant funding, and a Prosperity Partnership. A.M.U. also received from EPSRC Centre for Doctoral Training (CDT) for Science and Applications of Plastic Electronic Materials financial support through a graduate studentship.

\section{Compliance with Ethical Standards}

Conflict of interest The authors declare that they have no conflict of interest.

Open Access This article is licensed under a Creative Commons Attribution 4.0 International License, which permits use, sharing, adaptation, distribution and reproduction in any medium or format, as long as you give appropriate credit to the original author(s) and the source, provide a link to the Creative Commons licence, and indicate if changes were made. The images or other third party material in this article are included in the article's Creative Commons licence, unless indicated otherwise in a credit line to the material. If material is not included in the article's Creative Commons licence and your intended use is not permitted by statutory regulation or exceeds the permitted use, you will need to obtain permission directly from the copyright holder. To view a copy of this licence, visit http://creativecommonshorg/licenses/by/4.0/.

\section{Appendix 1: The Effects of Charge-Carrier Scattering Time}

\subsection{Assessment of Photoconductivity Estimates}

In Section 3 of the main text, we validate our formula (Eq. 17) using the example of a thin film, exhibiting Drude-type photoconductivity with very short charge-carrier scattering time $(\tau \rightarrow 0)$. The conductivity of the charge carriers in such a semiconductor is purely real, and the only imaginary contribution to the apparent conductivity originates from the dielectric constant $\epsilon_{L}=10$ (see Eq. 7).

Here, we test the validity of our approximation for a range of charge-carrier scattering times, to investigate the influence of the imaginary conductivity on the accuracy of our expression for photoconductivity. Figure 7 (c, d, g, h) shows the relative errors of the calculated real part of the photoconductivity, together with Drude conductivity spectra (a, b, e, f) used in the calculations. We note that for the large 
a
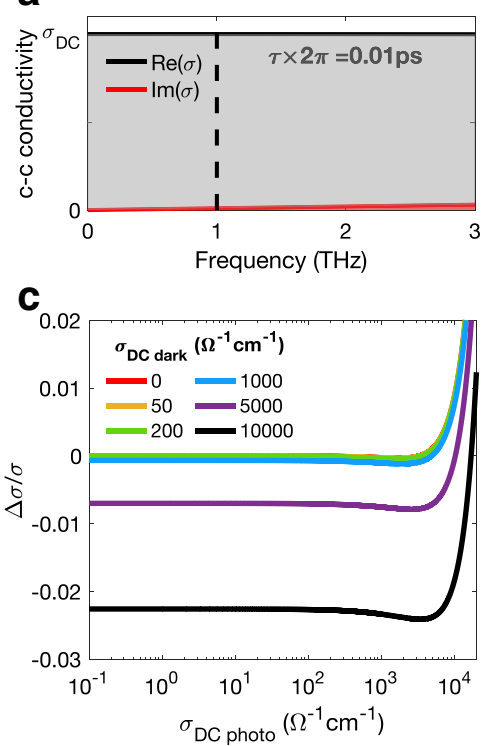

e

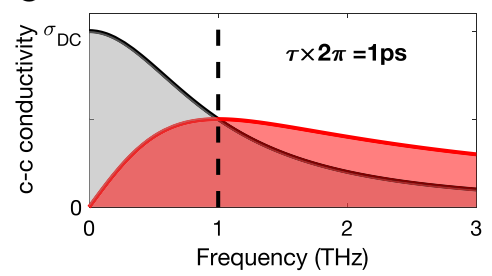

g

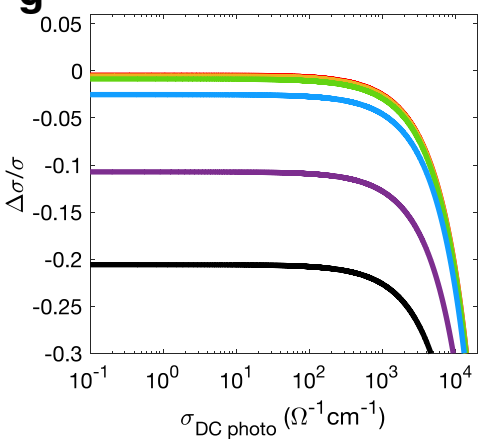

b

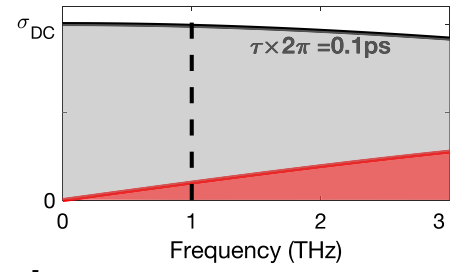

d

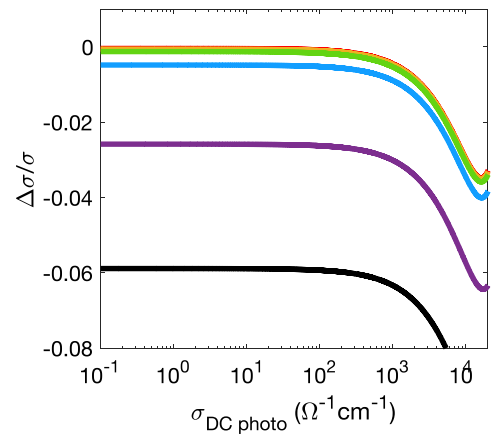

f

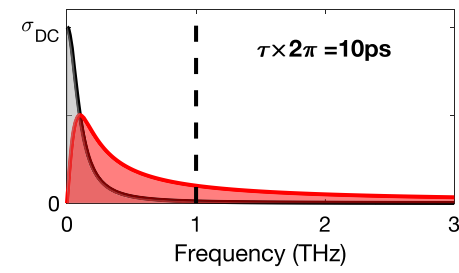

h

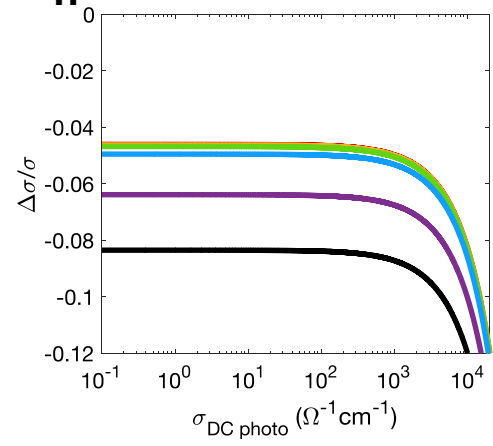

Fig. 7 The effect of charge-carrier scattering time and dark conductivity value on the error of calculated photoconductivity. $\mathbf{a}, \mathbf{b}, \mathbf{e}, \mathbf{f}$ Drude-type conductivity spectra of charge-carriers and $\mathbf{c}, \mathbf{d}, \mathbf{g}, \mathbf{h}$ the corresponding relative errors of photoconductivity calculated from Eq. 17 are shown for thin films with different charge-carrier scattering times, varying between $2 \pi \tau=0.01$ and $2 \pi \tau=10 \mathrm{ps}$. The relative error of the real part of photoconductivity, $\Delta \sigma / \sigma=\left(\sigma_{\text {photo }}-\sigma_{\text {calculated }}\right) / \sigma_{\text {photo }}$, was calculated at frequency of $1 \mathrm{THz}$ (marked with dotted line in the Drude conductivity spectra), for 400-nm-thick thin films with dark DC chargecarrier conductivities indicated by the legend. A dielectric constant of $\epsilon_{L}=10$ was used in the absence of charge carriers. The errors are shown as functions of the DC conductivity of photoexcited charge carriers, which exhibit the same Drude conductivity as the background carriers introduced by doping 
Fig. 8 Simulated time-domain transmission function (black solid line) through 400-nm-thick thin films with Drude conductivity, shown for chargecarrier scattering times of

a $2 \pi \tau=0.1 \mathrm{ps}, \mathbf{b} 2 \pi \tau=0.5 \mathrm{ps}$, c $2 \pi \tau=1$ ps and $\mathbf{d} 2 \pi \tau=2$ ps. The black dotted line represents the experimental transmission through bare quartz, used to calculate the time-domain field transmitted through the sample. The insets show the complex conductivity of the sample used in the calculations. In the insets, the blue straight line represents the real conductivity, estimated using $-\Delta T /(T+\Delta T)$ value from the peak of the $\mathrm{THz}$ pulse. The black dotted line shows the averaged value of the real part of conductivity, weighted by power spectrum of the terahertz pulse used in the experiment. The lattice contribution to the dielectric response of $\epsilon_{\mathrm{L}}=10$ was used for all samples
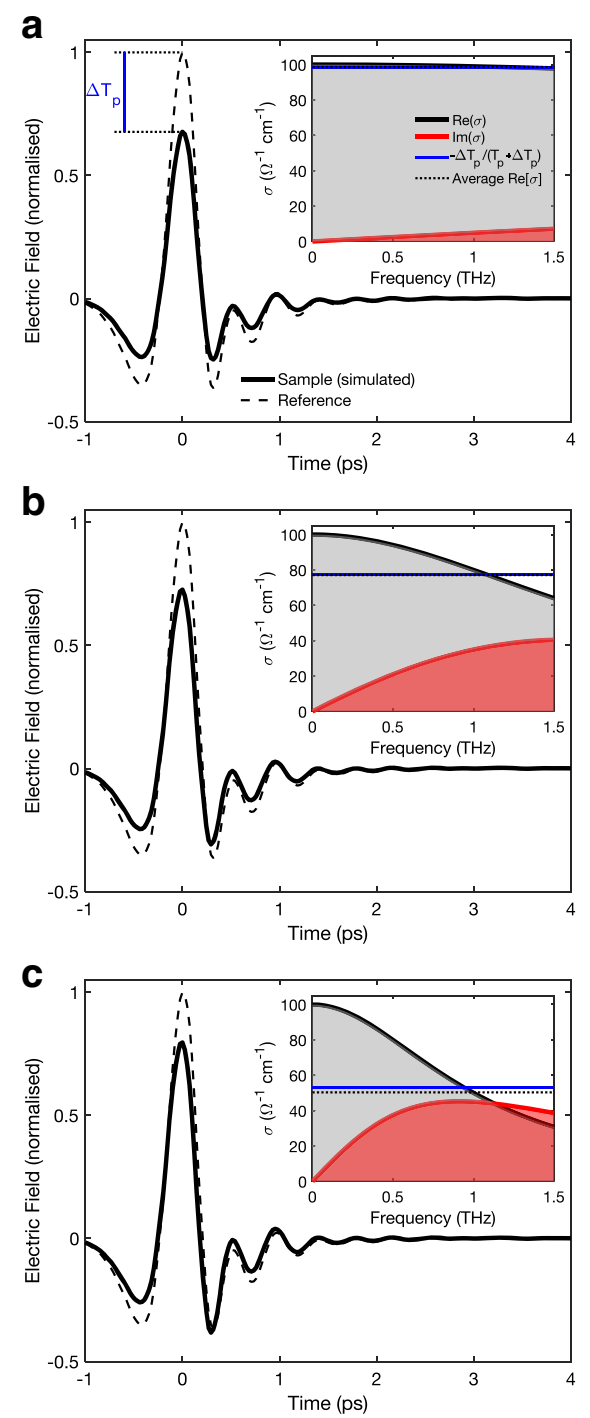

d

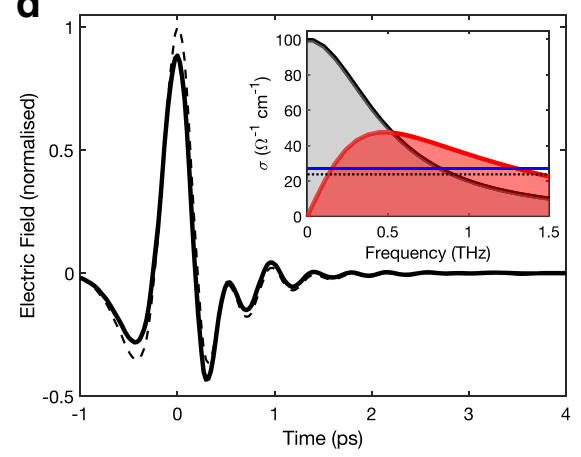


range of charge-carrier scattering times, spanning 4 orders of magnitude, our approximated expression for photoconductivity provides an accurate value of its real part up to a total conductivity of $\sim 10^{3} \Omega^{-1} \mathrm{~cm}^{-1}$. Additionally, for very short scattering times, $2 \pi \tau<0.1 \mathrm{ps}$, we note that the expression is valid for dark- and photoconductivity values up to $\sim 10^{4} \Omega^{-1} \mathrm{~cm}^{-1}$, above which the assumption of negligible optical thickness breaks down (as discussed in Section 3.1).

These findings imply that our approach can be used for a range of doped semiconducting thin films, with large background charge-carrier densities and various charge-carrier scattering rates, making it extremely useful for investigating photoexcited charge-carrier dynamics of novel doped semiconducting materials.

\subsection{Simulated Transmission Functions}

Section 4 outlines a method of obtaining the real part of the dark conductivity from the peak of the time-domain $\mathrm{THz}$ pulse, using the example of perovskite thin films, which show relatively flat spectral response due to short charge-carrier scattering times. We evaluate this method of estimating the dark conductivity for thin films with various conductivity spectra, by simulating the time-domain transmission through thin films and comparing the value obtained from the peak of the pulse to the DC Drude conductivity. Figure 8 shows the calculated time-domain transmissions and the spectra used in the simulations. We find that the real conductivity extracted from the time-domain peak follows closely the spectral average (weighted by the power spectrum of THz pulse) of the real part of conductivity, but agrees with the DC conductivity only for the case of short charge-carrier scattering time $(2 \pi \tau<0.1 \mathrm{ps})$. For semiconductors with longer scattering times, this method leads to an underestimate of the DC conductivity value. Therefore, full spectral analysis of the photoconductivity is necessary to obtain the charge-carrier mobility in materials with long charge-carrier scattering time, although a time-dependent measurement of the peak's intensity (1D OPTP measurement) would still result in accurate estimates of charge-carrier decay dynamics.

\section{References}

1. National Renewable Energy Laboratory, NREL Efficiency Chart (accessed May 29, 2020).

2. K. Chopra, P. Paulson, V. Dutta, Progress in Photovoltaics: Research and applications 12(2-3), 69 (2004).

3. S.D. Stranks, G.E. Eperon, G. Grancini, C. Menelaou, M.J. Alcocer, T. Leijtens, L.M. Herz, A. Petrozza, H.J. Snaith, Science 342(6156), 341 (2013).

4. M.A. Green, A. Ho-Baillie, H.J. Snaith, Nature photonics 8(7), 506 (2014).

5. Q. Lin, A. Armin, R.C.R. Nagiri, P.L. Burn, P. Meredith, Nature Photonics 9(2), 106 (2015).

6. C. Wehrenfennig, G.E. Eperon, M.B. Johnston, H.J. Snaith, L.M. Herz, Advanced materials 26(10), 1584 (2014).

7. C. Wehrenfennig, M. Liu, H.J. Snaith, M.B. Johnston, L.M. Herz, Energy \& Environmental Science 7(7), 2269 (2014).

8. C.S. Ponseca Jr, T.J. Savenije, M. Abdellah, K. Zheng, A. Yartsev, T. Pascher, T. Harlang, P. Chabera, T. Pullerits, A. Stepanov, et al., Journal of the American Chemical Society 136(14), 5189 (2014). 
9. W. Rehman, R.L. Milot, G.E. Eperon, C. Wehrenfennig, J.L. Boland, H.J. Snaith, M.B. Johnston, L.M. Herz, Advanced Materials 27(48), 7938 (2015).

10. G.R. Yettapu, D. Talukdar, S. Sarkar, A. Swarnkar, A. Nag, P. Ghosh, P. Mandal, Nano letters 16(8), 4838 (2016).

11. L.M. Herz, ACS Energy Letters 2(7), 1539 (2017).

12. J. Lloyd-Hughes, T.I. Jeon, Journal of Infrared, Millimeter, and Terahertz Waves 33(9), 871 (2012).

13. H.K. Nienhuys, V. Sundström, Physical Review B 71(23), 235110 (2005).

14. C. La-o-vorakiat, L. Cheng, T. Salim, R.A. Marcus, M.E. Michel-Beyerle, Y.M. Lam, E.E. Chia, Applied Physics Letters 110(12), 123901 (2017).

15. R. Ulbricht, E. Hendry, J. Shan, T.F. Heinz, M. Bonn, Reviews of Modern Physics 83(2), 543 (2011).

16. L. Duvillaret, F. Garet, J.L. Coutaz, IEEE Journal of selected topics in quantum electronics 2(3), 739 (1996).

17. J. Neu, K.P. Regan, J.R. Swierk, C.A. Schmuttenmaer, Applied Physics Letters 113(23), 233901 (2018).

18. W. Withayachumnankul, J.F. O'Hara, W. Cao, I. Al-Naib, W. Zhang, in 2014 39th International Conference on Infrared, Millimeter, and Terahertz waves (IRMMW-THz) (IEEE, 2014), pp. 1-2.

19. J. Jo, E. Jung, J.C. Park, J. Hwang, Current Applied Physics 20(2), 237 (2020).

20. J. Neu, C.A. Schmuttenmaer, Journal of Applied Physics 124(23), 231101 (2018).

21. T. Wang, M. Zalkovskij, K. Iwaszczuk, A.V. Lavrinenko, G.V. Naik, J. Kim, A. Boltasseva, P.U. Jepsen, Optical Materials Express 5(3), 566 (2015).

22. J.F. O'Hara, W. Withayachumnankul, I. Al-Naib, Journal of Infrared, Millimeter, and Terahertz Waves 33(3), 245 (2012).

23. M. Tinkham, Physical Review 104(3), 845 (1956).

24. R. Glover III, M. Tinkham, Physical Review 108(2), 243 (1957).

25. C.L. Davies, J.B. Patel, C.Q. Xia, L.M. Herz, M.B. Johnston, Journal of Infrared, Millimeter, and Terahertz Waves 39(12), 1236 (2018).

26. T.I. Jeon, D. Grischkowsky, Applied Physics Letters 72(23), 3032 (1998).

27. K. Willis, S. Hagness, I. Knezevic, Applied Physics Letters 102(12), 122113 (2013).

28. F. Yan, E.P. Parrott, B.S.Y. Ung, E. Pickwell-MacPherson, The Journal of Physical Chemistry C 119(12), 6813 (2015).

29. M.A. Ordal, R.J. Bell, R.W. Alexander, L.L. Long, M.R. Querry, Applied optics 24(24), 4493 (1985).

30. D.X. Zhou, E.P. Parrott, D.J. Paul, J.A. Zeitler, Journal of Applied Physics 104(5), 053110 (2008).

31. P. Tiwana, P. Parkinson, M.B. Johnston, H.J. Snaith, L.M. Herz, The Journal of Physical Chemistry C 114(2), 1365 (2010).

32. S. Sarkar, V.K. Ravi, S. Banerjee, G.R. Yettapu, G.B. Markad, A. Nag, P. Mandal, Nano letters 17(9), 5402 (2017).

33. D. Zhao, E.E. Chia, Advanced Optical Materials 8(3), 1900783 (2020).

34. M.G. Burdanova, A.P. Tsapenko, D.A. Satco, R. Kashtiban, C.D. Mosley, M. Monti, M. Staniforth, J. Sloan, Y.G. Gladush, A.G. Nasibulin, J. Lloyd-Hughes, ACS Photonics 6(4), 1058 (2019).

35. R.L. Milot, M.T. Klug, C.L. Davies, Z. Wang, H. Kraus, H.J. Snaith, M.B. Johnston, L.M. Herz, Advanced Materials 30(44), 1804506 (2018).

36. N.K. Noel, S.D. Stranks, A. Abate, C. Wehrenfennig, S. Guarnera, A.A. Haghighirad, A. Sadhanala, G.E. Eperon, S.K. Pathak, M.B. Johnston, et al., Energy \& Environmental Science 7(9), 3061 (2014).

37. M.H. Kumar, S. Dharani, W.L. Leong, P.P. Boix, R.R. Prabhakar, T. Baikie, C. Shi, H. Ding, R. Ramesh, M. Asta, et al., Advanced Materials 26(41), 7122 (2014).

Publisher's Note Springer Nature remains neutral with regard to jurisdictional claims in published maps and institutional affiliations. 